\title{
Physical Detection of Heteroduplexes during Meiotic Recombination in the Yeast Saccharomyces cerevisiae
}

\author{
DILIP K. NAG AND THOMAS D. PETES* \\ Department of Biology, The University of North Carolina, \\ Chapel Hill, North Carolina 27599-3280
}

Received 7 September 1992/Returned for modification 16 November 1992/Accepted 14 January 1993

\begin{abstract}
We describe a general physical method for detecting the heteroduplex DNA that is formed as an intermediate in meiotic recombination in the yeast Saccharomyces cerevisiae. We use this method to study the kinetic relationship between the formation of heteroduplex DNA and other meiotic events. We show that strains with the rad50, but not the rad52, mutation are defective in heteroduplex formation. We also demonstrate that, although cruciform structures can be formed in vivo as a consequence of heteroduplex formation between DNA strands that contain different palindromic insertions, small palindromic sequences in homoduplex DNA are rarely extruded into the cruciform conformation.
\end{abstract}

The exchange of DNA strands between interacting chromosomes, the formation of a heteroduplex, is a central feature of homologous recombination. In the yeast Saccharomyces cerevisiae, the first clear evidence that heteroduplexes were formed during meiotic recombination was based on classical genetic analysis (8). In a diploid strain heterozygous at a single locus (alleles $\mathrm{A}$ and a), although most tetrads segregate 2A:2a spores, two types of aberrant segregants are detected: (i) gene conversion events $(3 \mathrm{~A}: 1 \mathrm{a}$ or $1 \mathrm{~A}: 3 \mathrm{a}$ segregation), found at a median frequency of about $5 \%$ (9), and (ii) postmeiotic segregation (PMS) events, found at a median frequency (for most alleles) of less than 1\% (9, 20). PMS events are signalled by a spore colony in which the two heterozygous alleles segregate (8). In general, in $S$. cerevisiae, the most common classes of PMS are those with a single PMS event $(9,20)$, either $2 \mathrm{~A}: 1 \mathrm{a}: 1 \mathrm{~A} / \mathrm{a}$ spore colonies (called a $5 \mathrm{~A}: 3 \mathrm{a}$ segregation) or $1 \mathrm{~A}: 2 \mathrm{a}: 1 \mathrm{~A} / \mathrm{a}$ spore colonies (3A:5a). To retain consistent nomenclature, we will describe gene conversion events as 6:2 or 2:6 events, and normal Mendelian segregation as $4: 4$.

Since a PMS event is the segregation of two markers from a spore containing one haploid genome, these events are most simply interpreted as reflecting heteroduplex formation between the two alleles, with failure to repair the resulting mismatch (12). Most meiotic gene conversion events in yeast cells are most simply interpreted as reflecting heteroduplex formation between the two alleles, followed by mismatch repair $(9,20)$. For most mutations, gene conversion events are much more frequent than PMS events, indicating that most mismatches are efficiently repaired, although mutations caused by the insertion of small palindromic oligonucleotides result in poorly repaired mismatches (19). Since PMS and gene conversion events are often associated with crossing over of flanking markers, in most models of recombination, the heteroduplex is an intermediate in the production of a crossover (20).

The genetic assay for heteroduplex formation in yeast cells has two limitations. First, since the assay involves sectoring of spore colonies, the kinetics of heteroduplex formation during meiosis cannot be examined. Thus, one cannot determine the timing of heteroduplex formation rel-

\footnotetext{
${ }^{*}$ Corresponding author.
}

ative to other types of meiotic events. Second, since viable spores are required to detect a PMS event, one cannot examine heteroduplex formation in meiotic mutants that fail to sporulate or that fail to yield viable spores. The obvious solution to these limitations is to use a physical assay for heteroduplex formation. Lichten et al. (16) developed a denaturing gel procedure to detect heteroduplexes formed at the $A R G 4$ locus during meiotic recombination. In this report, we describe a simple general method of detecting heteroduplexes. We use this method to examine the timing of heteroduplex formation relative to other meiotic events and the properties of several mutants that affect meiotic recombination.

For our studies, we analyzed the HIS4 locus, which has an unusually high level of meiotic recombination in our genetic background $(5,6,19,31)$. At two other recombination hotspots in yeast cells, $A R G 4(28)$ and an ectopically located $L E U 2$ locus (4), meiosis-specific double-strand breaks have been observed. In this study, in addition to examining the formation of heteroduplexes at HIS4, we also found evidence for a double-strand break.

\section{MATERIALS AND METHODS}

Construction of plasmids. The plasmid pDN9 was constructed by cloning a XhoI-BglII fragment of the HIS4 gene in BamHI-SalI-treated YIp5 (19). The plasmid pDN11 was constructed by allowing the palindromic oligonucleotide DNO14 to self-anneal and then inserting the annealed product into the SalI site of pDN9; this SalI site is within the HIS4 coding sequence (Fig. 1A). The plasmid pDN35 was constructed comparably with the oligonucleotide DNO16. The oligonucleotide DNO14 is 32 bases long ( $5^{\prime}$ TCGAG TACTGTATGTGGATCCACATACAGTAC) and results in a frameshift mutation in HIS4 (his4-IR16). The oligonucleotide DNO16 is 30 bases long ( $5^{\prime}$ TCGAGCGTGTCTATC TGCAGATAGACACGC) and results in the in-frame mutation his4-IR15. The plasmid pSP3, a gift from S. Porter (University of North Carolina), has the DNO14 oligonucleotide inserted within the SalI site of the BIKl gene which is located on a XhoI fragment inserted at the SalI site of the YIp5 derivative B142. The plasmid pNKY349, provided by N. Kleckner (Harvard University), has a rad50S mutation marked with $U R A 3$ inserted in a pSP65-derived vector (1). 

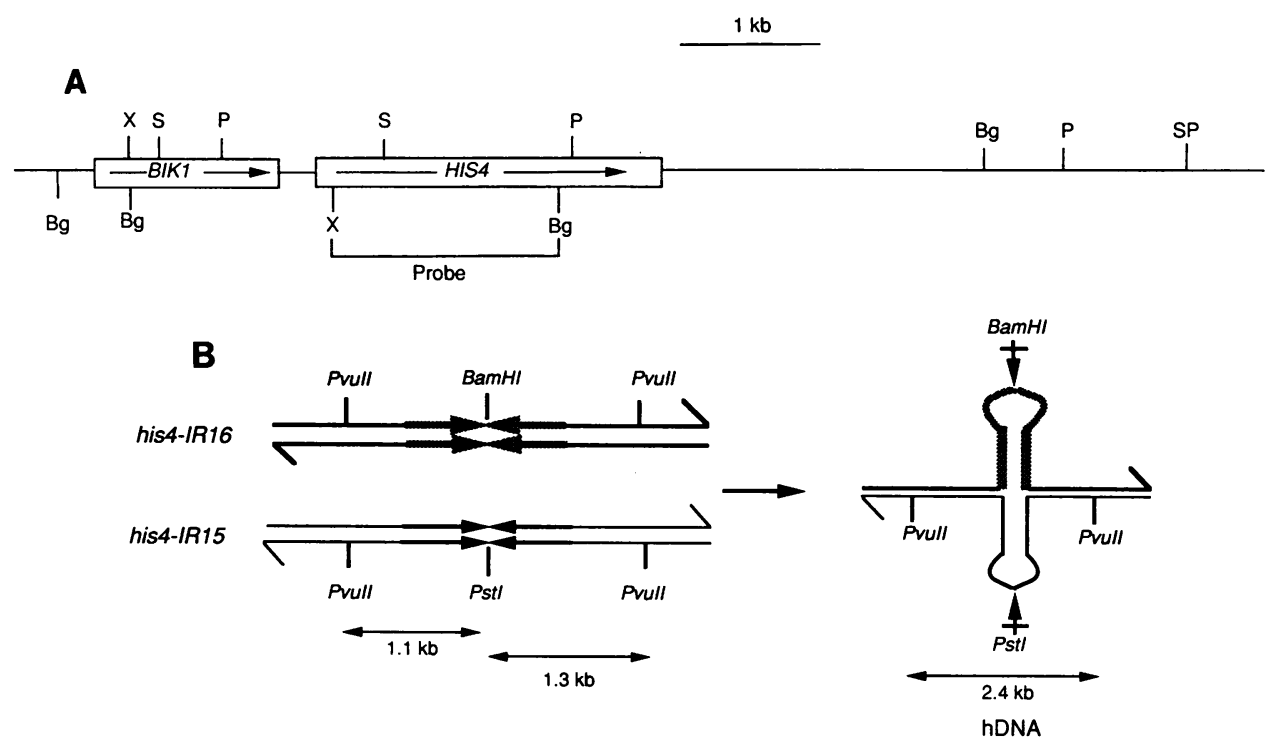

FIG. 1. Restriction sites relevant to the detection of crossovers and heteroduplexes. (A) Partial restriction map of the HIS4-BIK1 region. The boxes indicate the coding regions of the genes, and arrows indicate the direction of transcription. The line under the restriction map represents the region used as a probe for Southern analysis as described in Materials and Methods. Abbreviations: $\mathrm{Bg}, \mathrm{Bgl \textrm {II }}$; $\mathrm{P}, \mathrm{PvuII}$; $\mathrm{S}$, SalI; X, XhoI; SP, SpeI. (B) Expected cruciform conformation resulting from strand exchange involving the his4-IR16 and his4-IR15 alleles. The DNA strands derived from the two alleles are indicated by lines of different thickness. Thick arrows in inverted orientation represent the palindromic insertions of the two alleles. Lines at the bottom represent the lengths of the DNA fragments generated after digestion of the sample with the three enzymes PvuII, PstI, and BamHI. Distances representing the sizes of the fragments are not drawn to scale. Bars within the arrowhead indicate the insensitivity of the site to that enzyme.

The plasmids pSM22 and pNKY83 contain null alleles of the rad52 and rad50 genes, respectively, in which the coding sequences are replaced by $\operatorname{URA3}(2,24)$. The plasmid pMW33 (constructed by M. White, University of North Carolina) contains the URA3 gene inserted into a SpeI site located about $4 \mathrm{~kb}$ downstream of HIS4. This region was cloned as a BglII fragment into the BamHI site of YIp5. The plasmid pDN42 contains a XhoI-BglII fragment containing most of HIS4 inserted into the BamHI-XhoI-treated Bluescript vector $\mathrm{pBSII} \mathrm{SK}(-)$.

Construction of yeast strains. Most of the yeast strains were derived from the haploid strain AS4 ( $\alpha$ trp1 arg4 tyr 7 ura3 ade6) or AS13 (a leu2 ura3 ade6) $(27,29)$ by transformation. The haploid strains were constructed by either two-step (abbreviated TST; 23) or one-step (abbreviated OST; 22) transplacement procedures as follows: DNY72 (TST of AS4 with pDN35), DNY75 (TST of AS13 with pDN35), DNY45 (TST of AS13 with pDN11), DNY84 (OST of DNY72 with pMW33), DNY85 (TST of DNY45 with pSP3), DNY88 (OST of DNY45 with pNKY83), DNY89 (OST of DNY72 with pNKY83), DNY91 (OST of DNY45 with pNKY349), DNY92 (OST of DNY72 with pNKY349), DNY100 (OST of DNY45 with pSM22), DNY101 (OST of DNY72 with pSM22), DNY107 (OST of AS4 with pNKY349), and DNY106 (OST of AS13 with pNKY349). All strains were confirmed by Southern analysis. The diploid strains were constructed as follows ( $\times$ signifying a mating): DNY78 (DNY72 $\times$ DNY75), DNY86 (DNY84 $\times$ DNY85), DNY90 (DNY88 × DNY89), DNY93 (DNY91 $\times$ DNY92), DNY102 (DNY100 × DNY101), DNY112 (AS4 × AS13), and DNY115 (DNY106 $\times$ DNY107). The diploid MS1 was previously constructed by mating an AS13 derivative (MW1) with the his4-Sal mutation to an AS4 derivative (PD23) with the his4-713 mutation (30). Haploid strains used for his4 allele testing were F98 (a his4-280 trp1) and F99 ( $\alpha$ his4-280 ade2).

Media and genetic techniques. The media utilized were standard (25). The presporulation medium had $0.5 \%$ yeast extract, $1 \%$ peptone, $0.17 \%$ yeast nitrogen base, $1 \%$ potassium acetate, $0.5 \%$ ammonium sulfate, and $0.05 \mathrm{M}$ potassium biphthalate ( $\mathrm{pH}$ was adjusted to 5.5).

For tetrad analysis, the diploid strain DNY86 was sporulated at $18^{\circ} \mathrm{C}$ and the tetrads were dissected onto rich medium. After 3 days of growth at $30^{\circ} \mathrm{C}$, the spore colonies were replica plated onto various types of omission media. The HIS4 gene has three functional domains (HIS4A, $H I S 4 B, H I S 4 C$ ), each responsible for a different enzymatic activity (13). The diploid strain DNY86 is heterozygous for his4-IR16 and his4-IR15, both mutant alleles representing insertions at the same restriction site in HIS4A. The his4IR16 allele is a frameshift mutation that eliminates all three enzymatic activities, whereas the his4-IR15 mutation eliminates only $H I S 4 A$ activity. To score these alleles, we mated spore colonies derived from DNY86 to tester strains (F98 and F99) that contained his4-280 (a his4C mutation).

Physical analysis of DNA from meiotic cells. Most of the methods for preparation of meiotic DNA were based on protocols provided by C. Goyon and M. Lichten (11). To prepare meiotic cells, we inoculated a single colony into $2 \mathrm{ml}$ of yeast extract-peptone-dextrose (YPD) liquid medium (32). After overnight growth at $30^{\circ} \mathrm{C}$, a $0.15-\mathrm{ml}$ inoculum of this culture was used to inoculate $250 \mathrm{ml}$ of presporulation medium. This culture was incubated at $30^{\circ} \mathrm{C}$ until the cell concentration was $2 \times 10^{7}$ to $3 \times 10^{7} / \mathrm{ml}$. The cells were then washed with $50 \mathrm{ml}$ of $1 \%$ potassium acetate and suspended in a total volume of $250 \mathrm{ml}$ of $1 \%$ potassium acetate (supplemented with one-fifth the normal concentration of the nutritional requirements) at a cell concentration of $2 \times 10^{7} / \mathrm{ml}$. 
The culture was incubated at $25^{\circ} \mathrm{C}$. Fifteen-milliliter aliquots were taken at different time intervals, fixed by the addition of $15 \mathrm{ml}$ of cold $\left(-20^{\circ} \mathrm{C}\right) 95 \%$ ethanol and $0.75 \mathrm{ml}$ of $0.5 \mathrm{M}$ EDTA, and stored at $-20^{\circ} \mathrm{C}$.

For DNA isolation, the cells were pelleted by centrifugation, washed once with $0.5 \mathrm{ml}$ of $10 \mathrm{mM}$ Tris (pH 8.0)-1 mM EDTA (TE), and then suspended in $0.5 \mathrm{ml}$ of lysis buffer (10 $\mathrm{mM} \mathrm{Na} \mathrm{HPO}_{4}, 10 \mathrm{mM}$ EDTA, $1 \mathrm{M}$ sorbitol). 2-Mercaptoethanol $(3 \mu \mathrm{l})$ and $5 \mu \mathrm{l}$ of $25-\mathrm{mg} / \mathrm{ml}$ Zymolyase $(20,000 \mathrm{~T})$ were added to this suspension, and the mixture was incubated at $37^{\circ} \mathrm{C}$ for $30 \mathrm{~min}$. The resulting spheroplasts were harvested by centrifugation and resuspended in $0.5 \mathrm{ml}$ of 50 mM EDTA. The spheroplasts were lysed by the sequential addition of $7.5 \mu \mathrm{l}$ of $20 \%$ sodium dodecyl sulfate and $10 \mu \mathrm{l}$ of $10-\mathrm{mg} / \mathrm{ml}$ proteinase $\mathrm{K}$; this mixture was incubated at $65^{\circ} \mathrm{C}$ for $30 \mathrm{~min}$. Two hundred microliters of $5 \mathrm{M}$ potassium acetate was added to each tube, and the mixture was kept on ice for $45 \mathrm{~min}$. The mixture was then centrifuged in the cold for $15 \mathrm{~min}$, and the supernatant was collected. The DNA in the supernatent was precipitated with isopropanol, and the resulting pellet was resuspended in $200 \mu$ l of TE containing $50 \mu \mathrm{g}$ of RNase per $\mathrm{ml}$. After incubation at $65^{\circ} \mathrm{C}$ for $15 \mathrm{~min}$, the DNA was reprecipitated and the pellet was washed once with $70 \%$ ethanol. After being washed, the pellet was dried and dissolved in $50 \mu$ l of TE.

For each meiotic time point, the isolated DNA was treated with a 25 -fold excess of the desired restriction enzymes, and the resulting fragments were separated on a $0.8 \%$ agarose gel. Standard Southern blot procedures were performed (17). A XbaI-XhoI fragment derived from pDN42 containing a $B g l$ II-XhoI fragment of HIS4 was used as a hybridization probe; the $X b a I$ site is derived from the polylinker in pDN42. The relative amount of DNA present as heteroduplex was determined by densitometric analysis of the autoradiographs. After very long exposures, a small amount of undigested DNA is detected in all lanes. For the physical analysis of crossovers, since the $21-\mathrm{kb}$ parental DNA fragment transferred poorly relative to the $11-\mathrm{kb}$ parental DNA fragment, we calculated the total amount of hybridization to the parental bands by doubling the amount of hybridization to the 11-kb fragment.

Commitment to meiotic recombination; return-to-growth experiment. Cells become committed to meiotic levels of recombination before they become committed to meiotic division (26). Commitment to meiotic recombination can be determined by transferring a diploid (containing heteroalleles for auxotrophic mutations) to sporulation medium and, at various time intervals, returning the cells to medium allowing vegetative growth of prototrophs. The diploid MS1 is heteroallelic for mutations in HIS4. We sporulated a culture of MS1 using the same conditions described for the physical analysis of heteroduplexes. Aliquots were collected at various times during meiosis, diluted, and analyzed on plates containing synthetic dextrose (SD) complete medium (to monitor cell viability) and SD medium lacking histidine (to detect meiotic recombination).

Assay for nuclear division (32). Aliquots $(1 \mathrm{ml})$ of the sporulating culture were collected at different time points. Cells were harvested by centrifugation and fixed in $0.5 \mathrm{ml}$ of $4 \%$ formadehyde at room temperature for $20 \mathrm{~h}$. The fixed cells were washed with $1 \mathrm{ml}$ of water and stained with $0.5 \mathrm{ml}$ of $1-\mu \mathrm{g} / \mathrm{ml} \mathrm{4',6-diamidino-2-phenylindole} \mathrm{(DAPI).} \mathrm{After} \mathrm{in-}$ cubation at room temperature for $5 \mathrm{~min}$, the cells were washed with water and resuspended in $1 \mathrm{ml}$ of water. The nuclei were visualized with a Nikon fluorescence microscope.

\section{RESULTS}

System for detection of heteroduplex DNA. If a heteroduplex is formed between two genes, one of which contains an insertion, the inserted sequences would be expected to be in a single-stranded conformation. With few exceptions, heterozygous insertion or deletion mutations in yeast cells show very little PMS, suggesting that these single-stranded regions are usually efficiently recognized by cellular mismatch repair systems $(9,20)$. We have previously shown that a mismatch resulting from a heteroduplex formed between a wild-type gene and a gene with a palindromic insertion leads to high frequencies of PMS (19). We interpret this result as indicating that the stem-loop or hairpin configuration expected for a heterozygous palindromic insertion in a heteroduplex is inefficiently recognized by cellular mismatch repair systems.

We constructed a diploid strain (DNY86) that had one mutation caused by insertion of a 30 -bp palindrome into the SalI site of HIS4 (his4-IR15) and a second mutation (on the opposite homolog) with a 32-bp palindromic insertion of unrelated sequence into the same restriction site in HIS4 (Fig. 1). A heteroduplex formed between strands derived from these two genes would be expected to form a cruciform (Fig. 1B). Another feature of the palindromic insertions is that each contained a different restriction site in its center of symmetry: his4-IR15 had a PstI site and his4-IR16 had a BamHI site. Since bases at the tip of a palindrome are unpaired, the restriction sites of the insertions in the cruciform configuration should not be substrates for either PstI or BamHI. Thus, if the cruciform structure shown in Fig. 1B is not well-recognized by the mismatch repair systems, one should be able to detect heteroduplex DNA as DNA fragments of the appropriate size that are resistant to cleavage by PstI and BamHI.

Genetic evidence that heteroduplex-induced cruciforms are poorly recognized by mismatch correction mechanisms. As described above, heterozygous palindromic mutations have high levels of PMS relative to gene conversion, indicating that hairpin structures are poorly repaired by mismatch correction systems. To determine whether the cruciform structure shown in Fig. $1 \mathrm{~B}$ is also poorly repaired, we examined the patterns of aberrant segregation at the HIS4 locus in DNY86.

The HIS4 protein has three enzymatic activities encoded by complementing regions within the gene called $H I S 4 A$, $H I S 4 B$, and HIS4C (13). Although both HIS4 genes in DNY86 contain mutations, since the his4-IR15 mutation eliminates only HIS4A activity, whereas his4-IRI6 eliminates all three enzymatic activities, by complementation testing (described in Materials and Methods), one can examine the segregation of the two mutations separately. To describe the tetrad analysis, we use the nomenclature established for eight-spored fungi (since many of the aberrant segregants are PMS events), and the first number of each class represents the number of spores with the his4-IR15 allele. For example, the $6: 2$ class represents tetrads with three his4-IR15 spore colonies and one his4-IR16 spore colony. Of 316 tetrads examined, we found $186: 2,122: 6,32$ $5: 3,323: 5,7$ aberrant $4: 4,37: 1,21: 7,2$ aberrant $6: 2$, and one deviant $5: 3$. Approximately $34 \%$ of the tetrads had an aberrant segregation at HIS4, and $72 \%$ of the aberrant segregants were PMS events. These numbers are very similar to those observed for strains that were heterozygous for single palindromic insertions at the SalI site. For example, we previously observed $35 \%$ aberrant segregation $(72 \%$ of the aberrant segregation events were PMS) in a strain 


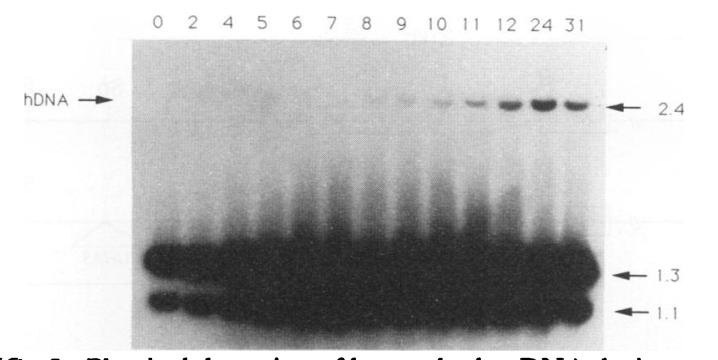

FIG. 2. Physical detection of heteroduplex DNA during meiosis. DNA samples isolated from meiotic cells (derived from the strain DNY86) were digested with PvuII, PstI, and BamHI. The resulting fragments were separated by gel electrophoresis and examined by Southern analysis with the region shown in Fig. 1A as a hybridization probe. The sizes of the fragments are indicated by arrows on the right, and the numbers on the top indicate the time (in hours) after the induction of meiosis. The 2.4-kb fragment represents the heteroduplex DNA (hDNA).

heterozygous for the his4-lop mutation (19). We conclude, therefore, that the cruciform structure shown in Fig. 1B, like the hairpin structure, is poorly recognized by the mismatch repair system.

Physical detection of heteroduplexes. As shown in Fig. 1A, most of the HIS4 gene and a portion of BIK1 are located on a 2.4-kb PvuII fragment. If the parental chromosomes of DNY86 are treated with PvuII, BamHI, and PstI, two fragments of 1.1 and $1.3 \mathrm{~kb}$ would be observed, since one insertion has a BamHI site and the other has a PstI site. If a heteroduplex involving the palindromic insertions is formed, then a band of $2.4 \mathrm{~kb}$ will also be observed. As described above, we have genetic evidence for heteroduplexes involving the palindromic insertions. To obtain supporting physical evidence, we transferred DNY86 to sporulation medium and collected cells at various time intervals. DNA was isolated from these cells and treated with PstI, BamHI, and PvuII. The resulting fragments were separated by gel electrophoresis, transferred to membranes, and hybridized to a probe containing sequences from the 2.4-kb PvuII fragment (Fig. 1A). A representative example of the time course of appearance of the heteroduplex DNA during meiosis is shown in Fig. 2. Heteroduplex DNA begins to appear at about $6 \mathrm{~h}$ after induction of meiosis and reaches a maximum level of about $6 \%$ of the total HIS4-specific DNA.

Since it is apparent from Fig. 2 that the $2.4-\mathrm{kb}$ band is meiosis specific, as expected for heteroduplex DNA, it is unlikely that this band represents partially digested DNA. However, the 2.4-kb band might reflect a cruciform extruded from a single chromatid (as shown in Fig. 3A) rather than a cruciform reflecting heteroduplex formation between two nonsister chromatids. To check this possibility, we constructed a diploid strain, DNY78, that was homozygous for the his4-IR15 mutant allele. If meiosis-specific intrachromatid cruciforms could form, one should see a 2.4-kb band. Since no such band was observed (Fig. 3B), we conclude that a 30-bp palindrome is rarely extruded into a cruciform in vivo; a similar conclusion was also reached from in vitro studies on the kinetics of cruciform formation (10). In addition, this result indicates that the 2.4-kb band observed in DNY86 represents heteroduplexes formed at the HIS4 locus. Additional data supporting this interpretation based on the analysis of $\mathrm{Rec}^{-}$mutants will be discussed below.

Kinetic relationship of heteroduplex formation with other meiotic events. The level of heteroduplex formation as a

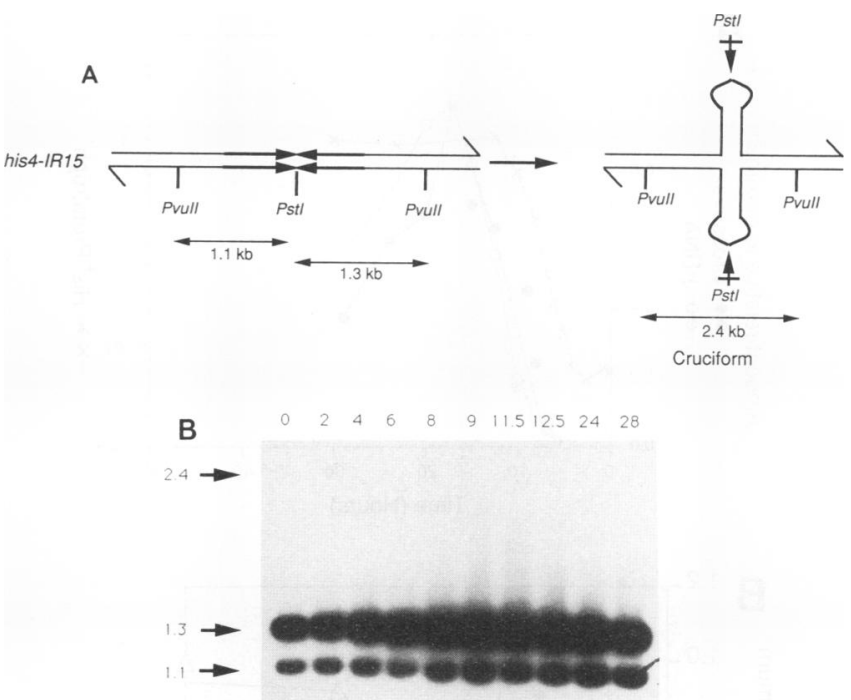

FIG. 3. Cruciform formation in homoduplexes. (A) Restriction fragments expected as a consequence of intramolecular cruciform formation in DNY78, a strain homozygous for the his4-IR15 mutation. If cruciforms were extruded from a homoduplex, one would expect a 2.4-kb fragment. (B) Southern analysis of DNA isolated from meiotic cells of DNY78. The DNA samples were treated with $P s t I$ and $P v u I I$. No fragment with the size expected for a cruciform extruded from a homoduplex $(2.4 \mathrm{~kb})$ was observed. Numbers on left show size in kilobases.

function of time in sporulation medium is shown in Fig. 4. Heteroduplex formation increases to a maximum of $6 \%$ of the total DNA at $16 \mathrm{~h}$ after induction of meiosis and then declines as spores are formed. As described in the Discussion, this decline is likely to reflect the difficulty of isolating meiotic DNA from spores. In addition to examining the timing of heteroduplex formation, we monitored several other landmark events in meiosis including commitment to meiotic recombination, formation of mature crossover products, meiotic nuclear divisions, and spore formation. These results are summarized in Fig. 4 and discussed in detail below.

(i) Commitment to meiotic recombination. Meiotic levels of recombination can be induced in diploid cells without commitment to meiotic divisions, if the cells are withdrawn from the sporulation medium and transferred to nutrient medium (26). Meiotic recombination is generally monitored with heteroallelic markers. In our experiments, we used the diploid MS1, which was heteroallelic at the HIS4 locus, and monitored meiotic recombination by plating the cells on medium lacking histidine. In general, heteroallelic recombination reflects gene conversion (20). As shown in Fig. 4, commitment to meiotic recombination begins at about $4.5 \mathrm{~h}$ after the induction of meiosis, and the maximum level of recombination is achieved at about $12 \mathrm{~h}$. The timing of commitment to meiotic levels of recombination appears to precede slightly heteroduplex formation, suggesting that some recombination events initiated during meiosis can result in heteroduplex formation in vegetative medium.

(ii) Timing of conversion and crossovers. As stated in the Introduction, in most models of recombination, the heteroduplex is an intermediate in the production of a reciprocal exchange. Thus, one would expect that formation of the heteroduplex should precede formation of the mature crossover. Crossovers can be monitored physically with strains containing heterozygous restriction sites (3). We constructed 

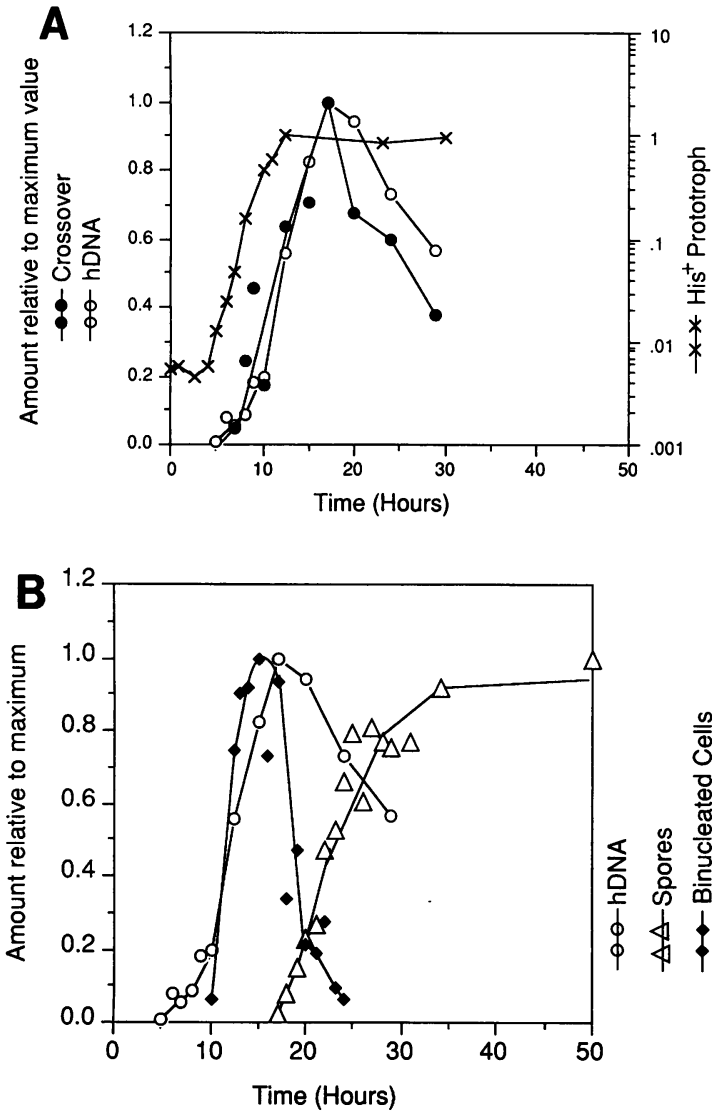

FIG. 4. Appearance of heteroduplex DNA relative to commitment to meiotic recombination, mature crossovers, binucleate cells (meiosis I), and spore formation. Commitment to meiotic recombination was measured by scoring $\mathrm{His}^{+}$prototrophs in return-togrowth experiments with the heteroallelic diploid MS1. All other measurements were done in single cultures of DNY86. Heteroduplex DNA and crossovers were assayed as described in the legends to Fig. 2 and 5, respectively. Spores in formaldehyde-fixed cells were counted under the microscope; two-, three-, and four-spored asci were counted. A total average of 200 cells were counted for each point. Binucleate cells were scored with the fluorescent stain DAPI, as described in Materials and Methods. Two graphs are used for clarity of presentation. (A) Heteroduplex formation relative to commitment to meiosis and crossovers. The levels of heteroduplexes and crossovers are shown as a fraction of the maximum observed level (indicated by the number 1 on the ordinate). The commitment data are shown on an exponential scale. The maximum observed values were $6 \%$ of total meiotic DNA for the heteroduplex DNA, 6 to $7 \%$ of total for the crossover fragment, and a frequency of $1.4 \times 10^{-2}$ ( His $^{+}$spores per total cells) for the commitment analysis. (B) Heteroduplex formation relative to formation of binucleate cells and spore formation. All data are shown as a fraction of the maximum observed levels $(6 \%$ of total meiotic DNA for the heteroduplexes, $8 \%$ for the frequency of binucleate cells, and $53 \%$ for the frequency of sporulated cells).

DNY86 with two heterozygous restriction site markers flanking the HIS4 region (Fig. 5A). One marker was an insertion of $U R A 3$ (disrupting a SpeI site) located about $4 \mathrm{~kb}$ downstream of HIS4. The other marker was a palindromic insertion (containing a $\mathrm{BamHI}$ restriction site) disrupting a SalI site in $B I K 1$, located about $1 \mathrm{~kb}$ upstream of HIS4. As shown in Fig. 5A, one of the expected crossover products is a HIS4-specific SalI-SpeI fragment of $7 \mathrm{~kb}$, a size smaller than either parental SalI-SpeI fragment. This product was
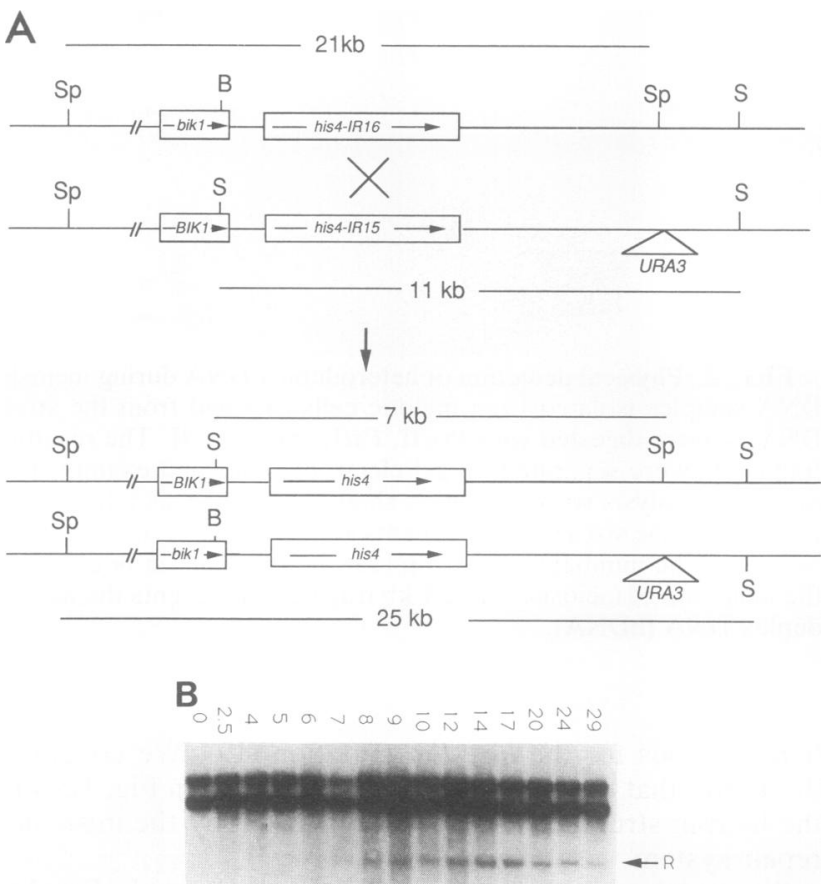

FIG. 5. Physical analysis of crossovers. (A) Restriction fragments expected as a consequence of crossing over in DNY86. The heterozygous sites and relevant homozygous sites on the two homologs are indicated. Any crossover between the heterozygous BIK1 SalI site (wild-type BIK1 gene with SalI site; mutant bik1 gene with an insertion of a palindromic oligonucleotide [with a BamHI site] that deletes the SalI site) and the heterozygous SpeI site (wild-type chromosome with SpeI site; other chromosome with insertion of $U R A 3$ that deletes this site) will produce crossover SalI-SpeI fragments of 7 and $25 \mathrm{~kb}$. The expected sizes of the parental fragments are 21 and $11 \mathrm{~kb}$. Abbreviations: Sp, SpeI; S, SalI; and B, BamHI. (B) Southern analysis of DNA (treated with SpeI and Sall) isolated from meiotic cells of DNY86. A XhoI-BglII fragment of the HIS4 coding region was used as a probe (Fig. 1A). The numbers on the top indicate the time points (in hours) of sample collection, and the recombinant band is indicated.

detected after about $6 \mathrm{~h}$ of sporulation (Fig. 5B) and had approximately the same kinetics of appearance as that observed for the heteroduplex band (Fig. 4A). As observed for heteroduplexes, the level of the 7-kb crossover (maximum level of 5 to $7 \%$ of total DNA) product declined as spores formed. As discussed below, we attribute this decline to a small fraction of cells that do not undergo meiosis. Gene conversion events also can result in the formation of a 7-kb recombinant band; in the Discussion, we calculate that crossovers and conversion events contributed approximately equally to formation of the 7-kb band.

(iii) Timing of meiosis I and sporulation. The meiosis I division was assayed by examining the frequency of binucleate cells by fluorescence microscopy. As shown in Fig. 4B, the maximum level of binucleate cells was observed between 12.5 and $17.5 \mathrm{~h}$. The broadness of this peak probably reflects a continued increase in the level of binucleate cells, coupled with loss of binucleate cells as a consequence of the second meiotic division. The kinetics of appearance of binucleated cells is similar to the kinetics of heteroduplex formation, presumably because of the asynchrony of the culture. After the premeiotic DNA replication, the homologs segregate to separate poles during the first meiotic division. 

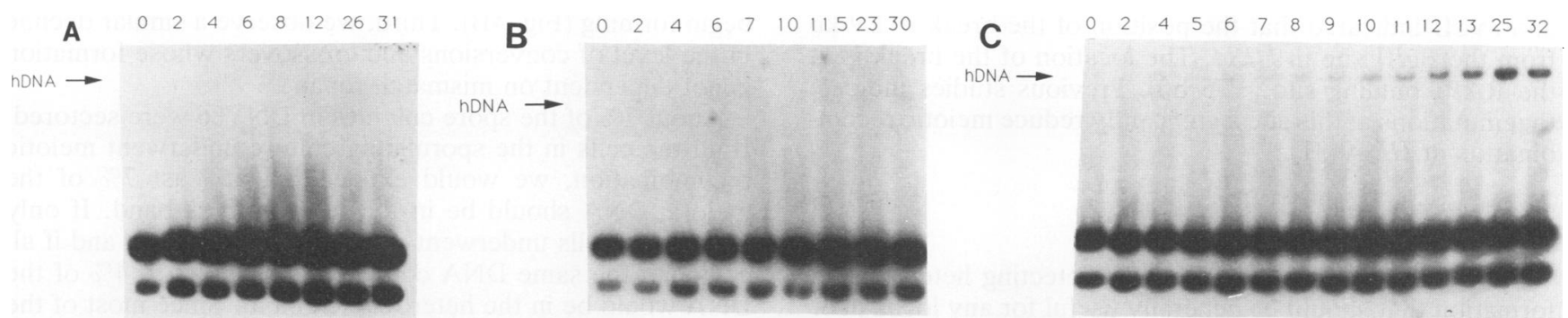

FIG. 6. Physical analysis of heteroduplex DNA in $\mathrm{Rec}^{-}$mutants. Southern analyses were performed as described in the legend to Fig. 2 . The numbers indicate the time in hours of sample collection after the induction of meiosis. The expected position of the heteroduplex DNA (hDNA) is indicated. (A) Diploid strain DNY90 (homozygous for rad50 null mutation); (B) diploid strain DNY93 (homozygous for rad50S mutation); (C) diploid strain DNY102 (homozygous for rad52 null mutation).

Spore formation begins at about $17 \mathrm{~h}$ after induction of meiosis, and the maximum level is reached at $32 \mathrm{~h}$ (Fig. 4B).

Heteroduplex formation in $\mathrm{Rec}^{-}$mutants. A large number of mutants have been isolated that reduce the frequency of meiotic recombination (reviewed in reference 20). These mutations greatly reduce spore viability, a result that is interpreted as indicating that recombination is necessary for proper chromosome disjunction at the first meiotic division (20). The spo13 mutation leads to bypass of the first meiotic division (14). The construction of strains with both the spo13 mutation and a $\mathrm{Rec}^{-}$mutation revealed that there are two classes of $\mathrm{Rec}^{-}$mutants, those that yield viable spores in combination with spo13 (class 1) and those that do not yield viable spores (class 2). The accepted interpretation of this result is that class 1 mutants represent early blocks in the recombination process (for example, formation of the DNA lesion that initiates recombination), whereas class 2 mutants affect later steps (for example, resolution of Holliday junctions).

It would be useful to determine by physical techniques the steps of recombination that are defective in the $\mathrm{Rec}^{-}$mutants. Two physical parameters that have been examined for some mutants are the ability to form mature crossovers and the ability to form a double-strand break. Borts et al. (3) showed that the rad6, rad52, and rad57 mutants were not defective in formation of mature crossovers but that the rad50 and spo11 mutants were. Since rad50 and spo11 were previously classified as class 1 mutants, and rad52 and rad57 were classified as class 2 mutants, these physical studies were consistent with genetic predictions. Cao et al. (4) found that rad50 null mutations prevented formation of a doublestrand break, whereas the rad50S mutation accumulated unprocessed double-strand breaks. An unprocessed break represents a double-strand break in which the broken ends have not been degraded into single-stranded tails. It has been suggested that these single-stranded ends are required for heteroduplex formation (28).

We examined heteroduplex formation in a rad50 null mutant, rad50S, and rad52 (Fig. 6). We found that the rad50 null mutant had no detectable heteroduplex. The rad50S mutant had a very small amount of heteroduplex (less than $10 \%$ wild-type levels). In contrast, strains with the rad52 mutation (Fig. 6C) had levels of heteroduplex formation that were only slightly (less than twofold) reduced relative to wild-type strains. In addition, heteroduplex formation was slightly delayed in the rad52 strain compared with the wild-type strain. This delay could reflect the slower growth rate of the rad52 strains.

In summary, our analysis of these mutants is consistent with earlier conclusions based on genetic and physical studies. The rad50 mutant is blocked before heteroduplex formation, and the rad52 mutant is blocked at some stage after heteroduplex formation. The absence of the 2.4-kb $P v u I I$ band in the rad50 strain also supports our conclusion that the presence of this band in wild-type strains does not represent a product of partial digestion with restriction endonucleases.

Double-strand breaks at HIS4. At two loci in $S$. cerevisiae, meiosis-specific double-strand breaks have been detected, and it has been suggested that these breaks represent the initiating lesion for meiotic recombination $(4,28)$. In our genetic background, despite the high level of HIS4 recombination, the level of double-strand breaks is low (Fig. 7A). We monitored the double-strand break by hybridizing a $H I S 4-s p e c i f i c$ probe to a BglII digest of meiotic DNA. There is a faint discrete band (about $1.9 \mathrm{~kb}$ in size) prior to sporulation. This band becomes more intense and more fuzzy during meiosis. In an isogenic rad50S strain, the band is more discrete (Fig. 7B); the maximum level of the band is $2.4 \%$ of the HIS4-specific DNA. Double digests with BglII

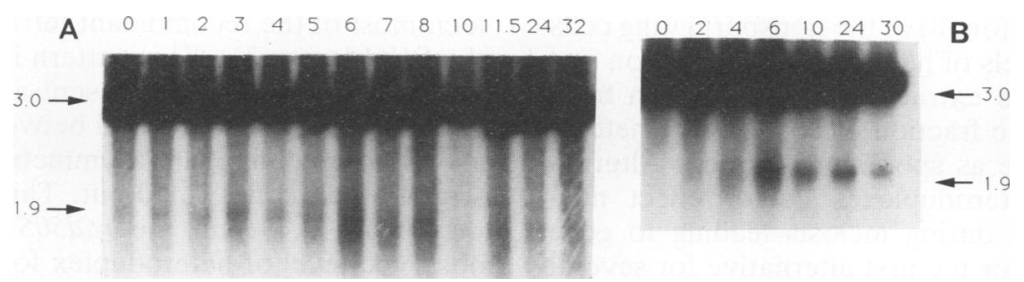

FIG. 7. Double-strand breaks at the HIS4 locus in wild-type and rad50S strains. DNA was isolated from cells after induction of meiosis and treated with BglII. The separated fragments were hybridized to the XhoI-BglII fragment of HIS4. The sizes of the fragments (in kilobases) are indicated. (A) Diploid DNY112 (homozygous HIS4 and RAD50); (B) diploid DNY115 (homozygous HIS4 and rad50S). 
and PvuII indicated that the position of the break is $1.9 \mathrm{~kb}$ from the BglII site in HIS4. The location of the break is at the RAP1 binding site $( \pm 75 \mathrm{bp})$. Previous studies indicate that mutations at this site significantly reduce meiotic recombination at HIS4 (31).

\section{DISCUSSION}

We describe above a method for detecting heteroduplex formation that should be generally useful for any locus in $S$. cerevisiae that has a high level of recombination. We used this method to examine the kinetic relationship between heteroduplex formation and other meiotic events and to examine heteroduplexes in several meiotic mutants. In addition, we found a meiosis-induced double-strand DNA break located upstream of the HIS 4 locus. The implications of these results are discussed below.

In our studies, commitment to heteroallelic recombination occurred before heteroduplex formation or formation of crossover products; the latter two events occurred at approximately the same time. In the crossover experiment, the 7-kb SalI-SpeI fragment was used as an indication of crossovers in the HIS4 region; approximately 5 to $7 \%$ of the total meiotic DNA was in this fragment. Gene conversion events involving either the heterozygous SalI or SpeI sites could also result in a 7-kb fragment. To obtain the 7-kb fragment by gene conversion, one would need either a $6: 2$ conversion event involving the $B I K 1$ marker or a $2: 6$ event involving the $U R A 3$ gene. The observed frequency of $6: 2$ conversion of the $B I K 1$ marker is $9 \%(21)$, and $6 \%$ of the tetrads had a $2: 6$ conversion for the SpeI::URA3 marker. Since only one spore of each tetrad is affected by these events, the expected fraction of meiotic chromosomes with a 7-kb fragment resulting from conversion is about $4 \%$. Thus, about $60 \%$ of the events that produced the 7-kb SalI-SpeI fragment were likely to be conversion events, and about $40 \%$ were crossovers.

The observation that heteroduplex formation occurred at approximately the same time as the conversions and crossovers has two interpretations. One interpretation is that heteroduplexes are an intermediate in the production of the other products and that this intermediate is quickly processed. Alternatively, it is possible that heteroduplexes and crossovers represent two different classes of recombination events. Intrachromosomal gene conversion events between repeated sequences are rarely associated with crossing over (15). In addition, some yeast mutations differentially affect gene conversion and crossing over (7). Since it is difficult to formulate a model of crossing over that does not require some level of heteroduplex, we prefer the first interpretation.

We observed a decline in the level of heteroduplexes late in meiosis. This decline can be explained in two ways. First, it may reflect an artifact of the difficulty in isolating DNA from spores. In our experiments, about $50 \%$ of the cells sporulate. If some fraction (or all) of the nonsporulating cells fail to undergo meiotic levels of heteroduplex formation and if DNA is more difficult to extract from spores than from unsporulated cells, then the fraction of DNA in the heteroduplex band would decline as spores are formed. Alternatively, the decline in heteroduplexes could reflect mismatches that are repaired during meiosis leading to gene conversion events. We favor the first alternative for several reasons. First, we find that the yield of DNA declines as spores are formed (data not shown). Second, the decline in heteroduplexes (Fig. 4A) occurs at the same time as spores begin forming (Fig. 4B). Third, we observe a similar decline in the level of conversions and crossovers whose formation is not dependent on mismatch repair.

About $7 \%$ of the spore colonies in DNY86 were sectored. If all the cells in the sporulating culture underwent meiotic recombination, we would expect that at least $7 \%$ of the meiotic DNA should be in the heteroduplex band. If only half of the cells underwent meiotic recombination and if all cells had the same DNA content, then only 3 to $4 \%$ of the DNA would be in the heteroduplex band. Since most of the unsporulated cells were unbudded, however, it is likely that the meiotic cells contain twice the amount of DNA as the unsporulated cells. Consequently, based on a $7 \%$ sectored colony frequency and assuming that only half of the cells underwent meiotic recombination, we calculate that about $5 \%$ of the DNA in DNY86 should be in heteroduplexes. This value is not significantly different from the observed level of heteroduplex $(6 \%)$.

Heteroduplex formation, as expected, precedes spore formation. Heteroduplex formation also initiates prior to meiosis I, although there is considerable overlap (presumably due to asynchrony) between cells that are initiating heteroduplexes and those that are completing meiosis I. C. Goyon and M. Lichten (11) have also performed experiments comparing the kinetics of heteroduplex formation (measured with denaturing gels) and other meiotic events. They find the same relative order of events at $A R G 4$ as we find at HIS4.

One advantage of physical methods of detecting recombination events is that it allows the analysis of recombination in strains that do not produce viable meiotic products. We found that rad50 mutants were defective in heteroduplex formation, whereas rad52 mutants had nearly wild-type levels of heteroduplex. These results are consistent with previous studies indicating rad50 mutants were blocked at an early step in recombination and rad52 mutants were blocked at a later stage (reviewed in reference 20). In addition, our results support earlier conclusions based on a physical analysis of crossover products (3).

As with several other meiotic recombination hotspots in yeast cells $(4,28)$, we have found evidence for a doublestrand break; in two previous studies $(11,33)$, a doublestrand break was also observed near HIS4. The band representing the broken chromosomes is diffuse in wild-type cells and discrete in the rad50S strain. The position of this break is close to the RAP1 binding site located between HIS4 and $B I K 1$. Previous studies indicate that the binding of the RAP1 protein to the RAP1 binding site is required for the highest level of meiotic recombination at both HIS4 and BIK1 (31).

There are several observations that indicate that this break may not represent the sole initiating lesion for recombination at HIS4. First, the break is not meiosis specific (Fig. 7A). Second, although genetic experiments indicate that the site located between HIS 4 and $B I K 1$ stimulates recombination at both loci $(6,31)$, in strains heterozygous for markers in both loci, most of the recombinant tetrads involve either HIS4 or the $B I K 1$ locus (21). This pattern indicates that the initiating lesion is not likely to represent a symmetrically processed double-strand break located between the two loci, although a model involving an asymmetrically processed doublestrand break is not ruled out. Third, the maximum level of the break $(2.4 \%)$ in the rad50S strains is less than the observed level of heteroduplex formation $(6 \%)$ in wild-type cells. This last conclusion has the caveats that we cannot rule out some processing of the double-strand break in the rad50S strains and we cannot be sure that the fraction of 
cells entering meiosis was identical in the wild-type and the rad50S strains.

Since heteroduplexes can be detected either by the method described above or by denaturing gels, one final issue is the comparative advantages of the two methods. For both methods, mutant alterations that lead to poorly repaired mismatches must be introduced into the locus of interest. We have found that palindromic insertions do not alter the pattern of recombination when inserted at a number of different positions in the HIS4 gene $(6,19)$. One disadvantage of the denaturing gel system is that the ease of detection of mismatches depends on the context of the mismatch (18). In addition, our system employs standard methods of gel analysis. One disadvantage of our method is that partially digested DNA products can be confused with heteroduplexes. Presumably, the relative advantages of the two methods will be clarified as other laboratories begin using these procedures.

In summary, we describe a general physical method of demonstrating heteroduplex formation in $S$. cerevisiae. We find that heteroduplex formation and mature crossovers occur at approximately the same time, and we show that heteroduplex formation is defective in rad50, but not rad52, mutant strains. Finally, we observe a double-strand break that occurs near the RAP1 binding site between HIS4 and BIK1.

\section{ACKNOWLEDGMENTS}

We thank G. Fink, N. Kleckner, M. Strand, S. Porter, M. White, and R. Schiestl for plasmids and yeast strains, E. Yeh and $I$. Schulman for their help in fluorescence microscopy, and members of the Petes laboratory for their comments on the manuscript. We thank M. Lichten for communicating unpublished data and protocols and for informing us of a double-strand break at HIS4. We appreciate the help of S. Porter and M. White in collecting cell samples.

This work was supported by National Institutes of Health Public Health Service grant GM24110.

\section{REFERENCES}

1. Alani, E., R. Padmore, and N. Kleckner. 1990. Analysis of wild-type and rad50 mutants of yeast suggests an intimate relationship between meiotic chromosome synapsis and recombination. Cell 61:419-436.

2. Alani, E., S. Subbiah, and N. Kleckner. 1989 . The yeast $R A D 50$ gene encodes a predicted $153-\mathrm{kD}$ protein containing a purine nucleotide-binding domain and two large heptad-repeat regions. Genetics 122:47-57.

3. Borts, R. H., M. Lichten, and J. E. Haber. 1986. Analysis of meiotic-defective mutations in yeast by physical monitoring of recombination. Genetics 113:551-567.

4. Cao, L., E. Alani, and N. Kleckner. 1990. A pathway for generation and processing of double-strand breaks during meiotic recombination in Saccharomyces cerevisiae. Cell 61:10891101.

5. Detloff, P., J. Sieber, J., and T. D. Petes. 1991. Repair of specific base pair mismatches formed during meiotic recombination in the yeast Saccharomyces cerevisiae. Mol. Cell. Biol. 11:737745.

6. Detlof, P., M. White, and T. D. Petes. 1992. Analysis of a gene conversion gradient at the HIS4 locus in Saccharomyces cerevisiae. Genetics 132:113-123.

7. Engebrecht, J., J. Hirsch, and G. S. Roeder. 1990. Meiotic gene conversion and crossing over: their relationship to each other and to chromosome synapsis and segregation. Cell 62:927-937.

8. Esposito, M. S. 1971. Post-meiotic segregation in Saccharomyces. Mol. Gen. Genet. 111:297-299.

9. Fogel, S., R. K. Mortimer, and K. Lusnak. 1981. Mechanisms of meiotic gene conversion, or "wanderings on a foreign strand," p. 289-339. In J. Strathern, E. W. Jones, and J. Broach (ed.), The molecular biology of the yeast Saccharomyces, vol. I. Cold Spring Harbor Laboratory, Cold Spring Harbor, N.Y.

10. Gellert, M., M. H. O'Dea, and K. Mizuuchi. 1983. Slow cruciform transitions in palindromic DNA. Proc. Natl. Acad. Sci. USA 80:5545-5549.

11. Goyon, C., and M. Lichten. Personal communication.

12. Holliday, R. 1964. A mechanism of gene conversion in fungi. Genet. Res. 5:282-304.

13. Keesey, J., R. Bigelis, and G. R. Fink. 1979. The product of the HIS4 gene cluster in Saccharomyces cerevisiae. J. Biol. Chem. 254:7427-7433.

14. Klapholz, S., and R. E. Esposito. 1980. Isolation of spo12-1 and spo13-1 from a natural variant of yeast that undergoes a single meiotic division. Genetics 96:567-588.

15. Klein, H. L. 1984. Lack of association between intrachromosomal gene conversion and reciprocal exchange. Nature (London) 310:748-753.

16. Lichten, M., C. Goyon, N. P. Schultes, D. Treco, J. W. Szostak, J. E. Haber, and A. Nicolas. 1990. Detection of heteroduplex DNA molecules among the products of Saccharomyces cerevisiae meiosis. Proc. Natl. Acad. Sci. USA 87:7653-7657.

17. Maniatis, T., E. F. Fritsch, and J. Sambrook. 1982. Molecular cloning: a laboratory manual. Cold Spring Harbor Laboratory, Cold Spring Harbor, N.Y.

18. Myers, R. M., T. Maniatis, and L. S. Lerman. 1987. Detection and localization of single base changes by denaturing gradient gel electrophoresis. Methods Enzymol. 155:501-527.

19. Nag, D. K., M. A. White, and T. D. Petes. 1989. Palindromic sequences in heteroduplex DNA inhibit mismatch repair in yeast. Nature (London) 340:95-98.

20. Petes, T., R. E. Malone, and L. S. Symington. 1991. Recombination in yeast, p. 407-521. In J. R. Broach, J. R. Pringle, and E. W. Jones, The molecular and cellular biology of the yeast Saccharomyces, vol. I. Cold Spring Harbor Laboratory, Cold Spring Harbor, N.Y.

21. Porter, S., M. A. White, and T. D. Petes. Unpublished data.

22. Rothstein, R. 1983. One-step gene disruption in yeast. Methods Enzymol. 101:202-211.

23. Scherer, S., and R. W. Davis. 1979. Replacement of chromosome segments with altered DNA sequences constructed in vitro. Proc. Natl. Acad. Sci. USA 76:4951-4955.

24. Schild, D., B. Konforti, C. Perez, W. Gish, and R. K. Mortimer. 1983. Isolation and characterization of yeast DNA repair genes. I. Cloning of the RAD52 gene. Curr. Genet. 7:85-92.

25. Sherman, F., G. R. Fink, and J. B. Hicks. 1982. Methods in yeast genetics. Cold Spring Harbor Laboratory, Cold Spring Harbor, N.Y.

26. Sherman, F., and H. Roman. 1963. Evidence for two types of allelic recombination in yeast. Genetics 48:255-261.

27. Stapleton, A., and T. Petes. 1991 . The Tn $3 \beta$-lactamase gene acts as a hotspot for meiotic recombination in yeast. Genetics 127:39-51.

28. Sun, H., D. Treco, and J. W. Szostak. 1991. Extensive 3'overhanging, single-stranded DNA associated with the meiosisspecific double-strand breaks at the $A R G 4$ recombination initiation site. Cell 64:1155-1161.

29. Symington, L. S., and T. D. Petes. 1988. Expansions and contractions of the genetic map relative to the physical map of yeast chromosome III. Mol. Cell. Biol. 8:595-604.

30. White, M. A., P. Detloff, M. Strand, and T. D. Petes. 1992. A promoter deletion reduces the rate of mitotic, but not meiotic, recombination at the HIS4 locus in yeast. Curr. Genet. 21:109116.

31. White, M. A., M. Wierdl, P. Detloff, and T. D. Petes. 1991. DNA-binding protein RAP1 stimulates meiotic recombination at the HIS4 locus in yeast. Proc. Natl. Acad. Sci. USA 88:97559759.

32. Yeh, E. Personal communication.

33. Zenvirth, D., T. Arbel, A. Sherman, M. Goldway, S. Klein, and G. Simchen. 1992. Multiple sites for double-strand breaks in whole meiotic chromosomes of Saccharomyces cerevisiae. EMBO J. 11:3441-3447. 